\title{
Non-uniform description of short CRLH Leaky Wave Antenna with the Generalized Array Factor Approach
}

\author{
Olivier Rance, Pierre Lemaitre-Auger, Romain Siragusa, and Etienne Perret \\ Université Grenoble Alpes - LCIS \\ 50 Barthélémy de Laffemas, 269002 Valence cedex 9, France \\ olivier.rance@lcis.grenoble-inp.fr
}

\begin{abstract}
Radiation-patterns of short uniform CRLH LWA of various lengths have been computed. They show differences from the "array factor approach" predictions which cannot be explained by this model. Better results are achieved if we consider non uniform distributions of the leakage and propagation constants along the line. Non uniform distributions are determined by an empirical method based on the generalized array factor. The origin of this phenomenon is discussed and compared to the case of small array antennas.
\end{abstract}

Keywords-CRLH LWA, array factor approach, mutual coupling.

\section{INTRODUCTION}

Leaky wave antennas (LWA) are known for more than seventy years now and are still attractive in some applications because of the directivity of the radiated beam and because of the simplicity with which they are feed [1]. In the beginning of the last decade, a new proposition of LWA, the Composite Right/Left Handed (CRLH) antenna [2], was made. It was a real technological breakthrough in LWA because broadside radiation became possible with LWA [3].

A CRLH LWA is a quasi-uniform LWA with periodical structure of period $p$ smaller than the guided wavelength $\lambda_{g}$. It is composed of series interdigital capacitor coupled with a shunt stub inductor that creates a unit cell. The cascading of several similar unit cells creates a transmission line in which a leaky mode propagates (when design for it). The simulation of such structures with electromagnetic simulators is, however, a cumbersome task that requires a lot of computing time. To remedy that problem, the array factor (AF) approach was proposed [4]. It is an efficient and a fast computation tool to properly predict the radiation pattern of any periodic LWA of arbitrary length, including CRLH LWA antennas. The basic idea is that each cell is assimilated to a point source antenna. This approach is particularly well suited for long antennas because the computation time is extremely small compared to full wave simulation with similar accuracy. On the other hand, when dealing with short CRLH LWA antennas (i.e. less than 20 units), the accuracy is less good: comparison between predicted radiation patterns and full wave simulation results do not perfectly match anymore [4-5].

We show in this paper that when the number of cells of a CRLH LWA decreases, the radiation pattern of the antenna becomes asymmetric and that asymmetry is theoretically impossible for a non tapered quasi-uniform LWA. To explain that, we make the hypothesis that the edge effects which are present at the beginning and at the end of the CRLH transmission line, results in a non-uniform distribution of the attenuation constant, $\alpha$, and of the phase constant, $\beta$, along the line. In other words, we assume here that a short CRLH LWA antenna must be considered like a tapered quasi-uniform LWA, even if it is made of identical unit cells. This is empirically shown with the use of the Generalized Array Factor Approach (GAFA) which is appropriate for such kind of LWA [6].

Part II of this paper shows that the classical uniform array factor approach cannot explained the dissymmetrical radiation patterns obtain with full wave simulations. In part III the assumption of non-uniform distribution of the electrical parameters is made. Finally an empirical technique based on the generalized $\mathrm{AF}$ is presented in order to determine the nonuniform distributions. A parallel with array antennas is made.

\section{LiMITATIONS OF THE UNIFORM MODEL}

\section{A. Empirical demonstration}

To illustrate the effect of short CRLH LWA on the radiation pattern, several antennas were designed with identical unit cells but with a different number of cells. However, the value of the input and output interdigital capacitors is twice the one of the inner interdigital capacitors because a symmetric layout was chosen. The layout of the unit cell used is the same as the one presented in [7]. It is balanced at $2.45 \mathrm{GHz}$ and match at $50 \Omega$. A prototype of a 13 cell CRLH LWA can be seen Fig. 1.

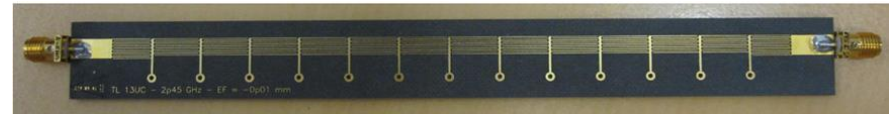

Fig. 1. 13 cell CRLH LWA. 


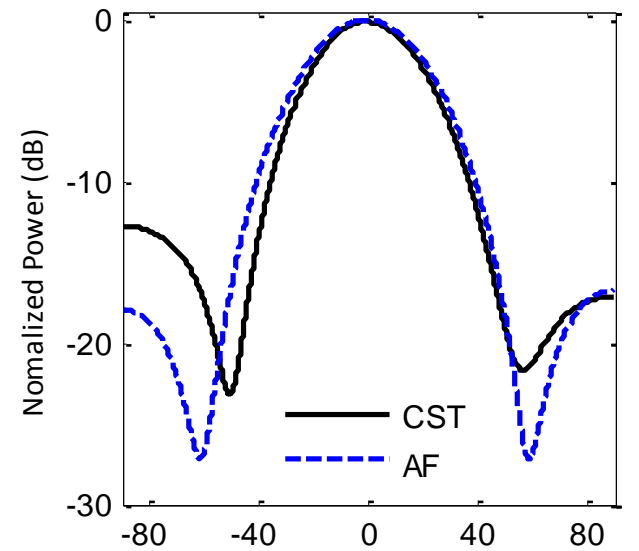

(a)
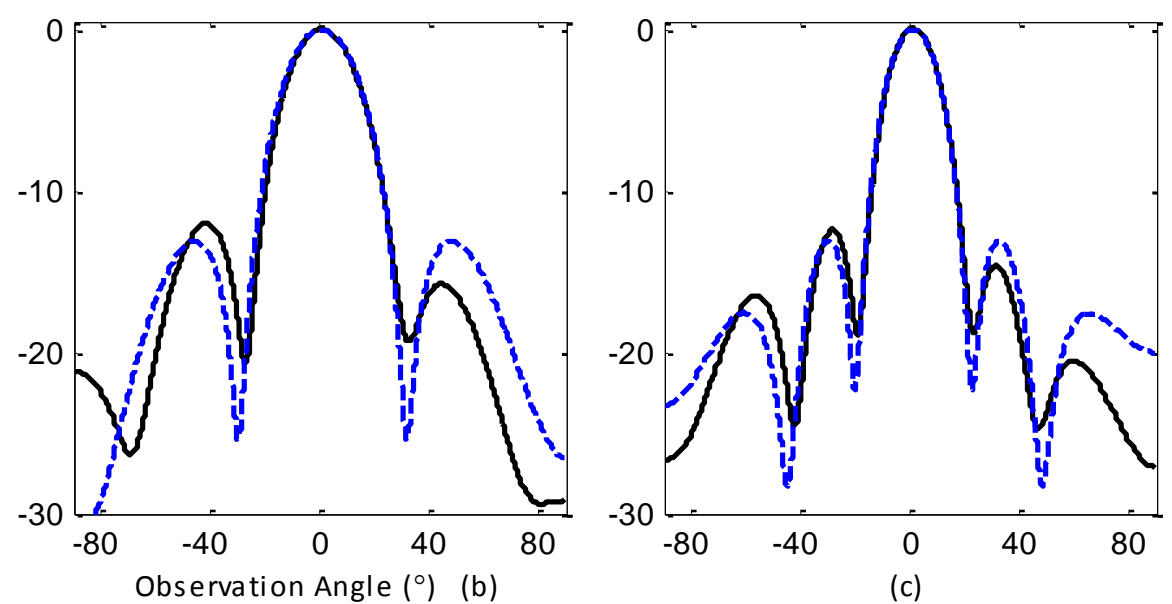

Fig. 2. Comparison between the radiation patterns obtained with the AF calculation and full-wave simulation. a)13 unit cells. b) 22 unit cells. c) 31 unit cells.

Each antenna was simulated with CST microwave studio to obtain the radiation pattern and the transmission coefficient, $S_{21}$. From that last simulated coefficient, average values of $\alpha$ and $\beta$ were extracted for each antenna with the help of [4]:

$$
\alpha=-\frac{\ln \left|S_{21}\right|}{L}, \quad \beta=-\frac{\varphi\left\{S_{21}\right\}}{L},
$$

where $L$ is the total length of the line. When using eq. (1), it is assumed that ohmic losses are negligible.

With the values of $\alpha$ and $\beta$ and with the array factor, radiation patterns are also computed [4]:

$$
A F(\theta)=\sum_{n=1}^{N} A_{n} e^{j \xi_{n}} \cdot e^{j(n-1) p k_{0} \sin \theta},
$$

where $(n-1) p$ denotes the position of the $n^{\text {th }}$ unit cell, $A_{n}$ and $\xi_{n}$ are the feeding amplitude and the phase respectively of the $n^{\text {th }}$ unit cell, $k_{0}$ is the wave number in the vacuum, and $\theta$ is the observation angle (i.e. the elevation angle). $A_{n}$ and $\xi_{n}$ are respectively given by $[4,8]$ :

$$
A_{n}=\sqrt{\alpha} A_{0} e^{-\alpha(n-1) p}, \quad \quad e^{j \xi_{n}}=e^{-j \beta(n-1) p} .
$$

The comparison of the radiation patterns obtained by simulation and with the AF for CRLH LWA of 13, 22 and 31 cells is represented in Fig.2. We clearly see a good agreement between the two for a 31 unit cell antenna for the main beam. However, the agreement is not so good for the levels of the sidelobes and it gets worst for decreasing number of unit cells. Also, for smaller number of unit cells, the electromagnetic simulation predict a thinner main beam that the AF calculation.

Another important observation that can be made is that the radiation patterns obtained with the electromagnetic simulations always show a dissymmetry between the levels of the sidelobes. For example, with 22 unit cells, we have $-12.1 \mathrm{~dB}$ for the left sidelobe and $-15.7 \mathrm{~dB}$ for the right one. Such a dissymmetry is present in the results published in $[4,5]$ but is not commented. However, this is not seen at all with the radiation pattern obtained by calculation with the $\mathrm{AF}$.

\section{B. Symmetry of the radiation diagram of a uniform $C R L H$ LWA}

In fact, with the AF approach, it can be shown that a CRLH LWA having a unique value of $\beta$ for all the unit cells must have a symmetric radiation pattern with respect to the main beam angle $\theta_{0}$.

Let's suppose that $\beta$ is constant for all the cells of a CRLH LWA antenna. $\beta$ is related to the main beam angle by:

$$
\beta=k_{0} \sin \theta_{0} .
$$

We define a normalized angular variable:

$$
u=k_{0} p\left(\sin \theta-\sin \theta_{0}\right) .
$$

The AF can then be expressed as a function of $u$. This leads to the expression of the radiation pattern, $g(u)$, which is proportional to the square of the AF:

$$
g(u)=|A F(u)|^{2}=\left|\sum_{n=1}^{N} A_{n} e^{j(n-1) u}\right|^{2}
$$

$g(u)=\left(\sum_{n=1}^{N} A_{n} \cos ((n-1) u)\right)^{2}+\left(\sum_{n=1}^{N} A_{n} \sin ((n-1) u)\right)^{2}$

From (7) it is evident that $g(u)$ is an even function of $u$. As a consequence, if $u_{0}$ is the coordinate of a sidelobe, the symmetric one corresponds to $-u_{0}$ and both sidelobes must have same values. We can notice that the parity of $g(u)$ is not a function of $A_{n}$ and, as such, is not related to the $\alpha$-distribution.

\section{HYPOTHESIS OF NON-UNIFORM $\beta$-PARAMETER SHORT CRLH LWA}

If we admit that the formalism of the AF should also be adequate for short CRLH LWA, then there are two explanations to the dissymmetry observed with electromagnetic simulations. 
First, one can consider that the radiation pattern of the unit cell, $S(\theta)$, is not purely isotropic and that it has to be included in the calculation of the diagram pattern. To check that idea, full-wave simulations of the unit cell have been realized to determine $S(\theta)$. They show a decay of $1.3 \mathrm{~dB}$ between the backfire and endfire directions which is not enough to explain the observations made.

The second possibility is to consider that the $\beta$-distribution along the line is not constant. From (1), this also implies that the $\alpha$-distribution is also not constant. A first indirect observation of that can be realized when observing the evolution of the extracted average values of $\alpha$ and $\beta$, for several CRLH LWA having a different number of cells. The Fig. 3 is an illustration of such an observation. $\alpha$ and $\beta$ are extracted from full-wave simulations with the help of (1). The two different curves show a logarithmic evolution and tend to stabilize around a constant value after 20 cells $\left(=1.8 \lambda_{0}\right)$. This initial evolution is not consistent with the idea that each cell has the same $\beta$-value. If it was the case, each cell would be a supplementary portion of the same transmission line. Thus, $\alpha$ and $\beta$ would remain unchanged.The extraction formulas (1) are global, i.e. they use the transmission parameter of the whole line. They can thus take into account a lot of different effects like inner reflection between adjacent cells or mutual coupling between the cells. The extraction formulas are based on the assumption that $\alpha$ and $\beta$ are constant along the line. However, if we use them on a tapered CRLH LWA ( $\alpha$ and $\beta$ not constant), it is easily shown that we obtain the average values.

All those observations leads to the conclusion that there is inside a CRLH LWA a non-uniform distribution of $\alpha$ and $\beta$. Physically, by analogy with antenna arrays, we can assume that there is mutual coupling between the cells. Those coupling can produce phase variations and impedance variations with a greater impact for close elements. For long lines, most of the elements will have the same environment and will therefore have the same values of $\alpha$ and $\beta$. Only few cells at the beginning and at the end of the line will have different values of $\alpha$ and $\beta$. The behavior of long CRLH LWA thus tends to the one of a pure non tapered LWA because the few elements near the edges do not contribute much to the radiation pattern. Naturally, for short CRLH LWA, the contribution of those elements becomes more important and the behavior of the antenna departs from the ideal one.

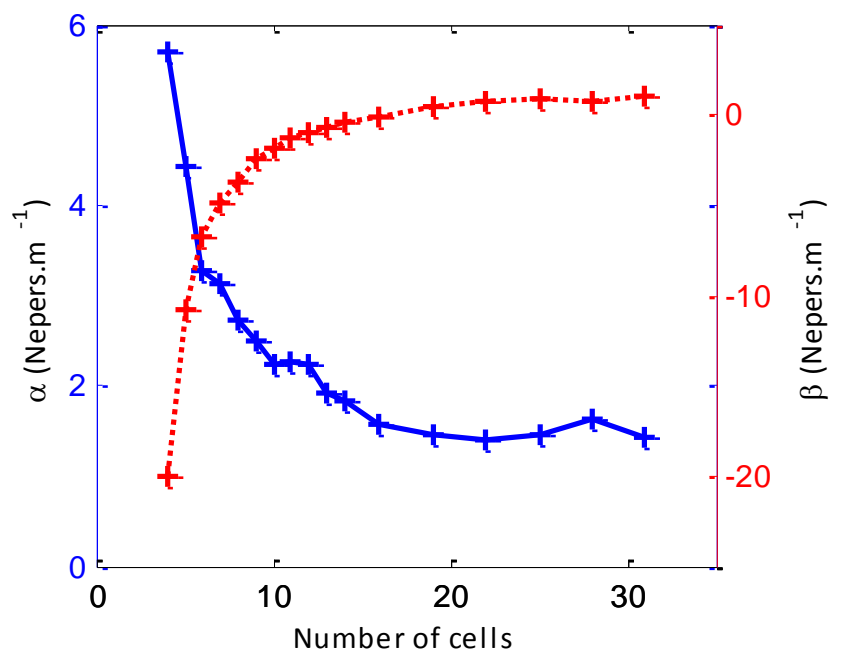

Fig. 3. Value of $\alpha$ and $\beta$ obtained from simulations for several CRLH line having a different number of cells extracted with (1).

\section{EMPERICAL MODELIZATION OF SHORT CRLH LWA}

To check that second hypothesis, we modeled short CRLH with a tapering function on $\alpha$ and $\beta$ even if all units cells are physically the same. The GAFA is used advantageously for that task [6]. Formula (3) is slightly modified to describe non uniform case:

$$
A_{n}=p \sqrt{\alpha_{n}} A_{0} e^{-p \sum_{i=1}^{n-1} \alpha_{i}}, \quad e^{j \xi_{n}}=e^{-j p \sum_{i=1}^{n-1} \beta_{i}},
$$

where the index $i$ and $n$ indicate that the value is relative to the $i^{\text {th }}$ and $n^{\text {th }}$ cell respectively.

For antenna arrays, the effect of mutual coupling can be evaluated straightforwardly thanks to the fact that each element can be fed separately. Techniques like mutual admittance matrix or incremental impedance are usually used to compensate the mutual coupling effects [9-10].

The leaky wave antenna feeding mechanism makes it impossible to isolate a single emitter and to use the same techniques. The method to model the non-uniform distribution of $\alpha$ and $\beta$ is then empirical in the present work.

Values of $\alpha$ and $\beta$ inside the different cells are considered as unknowns of the GAFA model. They are adjusted with a least square minimization to best fit the full wave radiation pattern. Unfortunately, this kind of numerical method can lead to
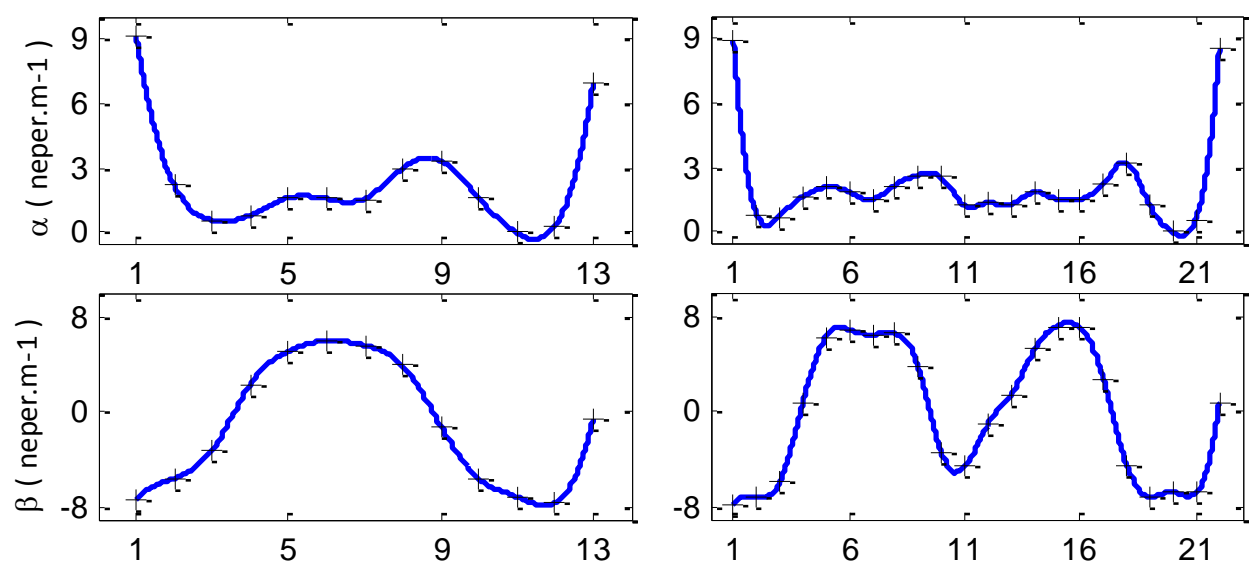

(a)
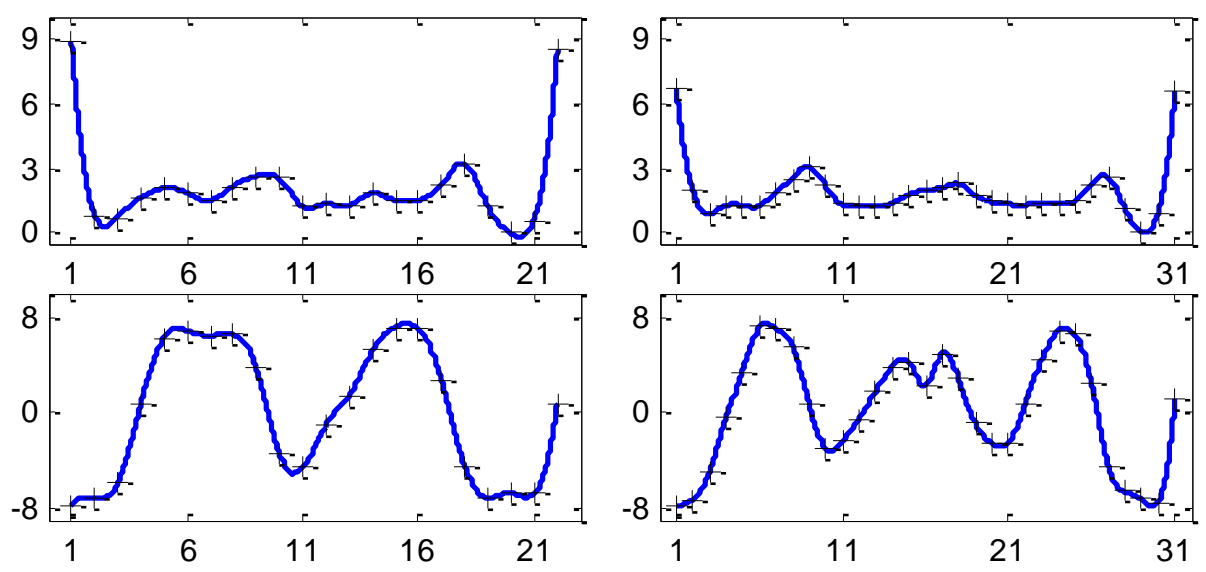

Cell Number

(b)

(c)

Fig. 4. Non uniform distribution of $\alpha$ and $\beta$. a) 13 unit cells. b) 22 unit cells. c) 31 unit cells. 


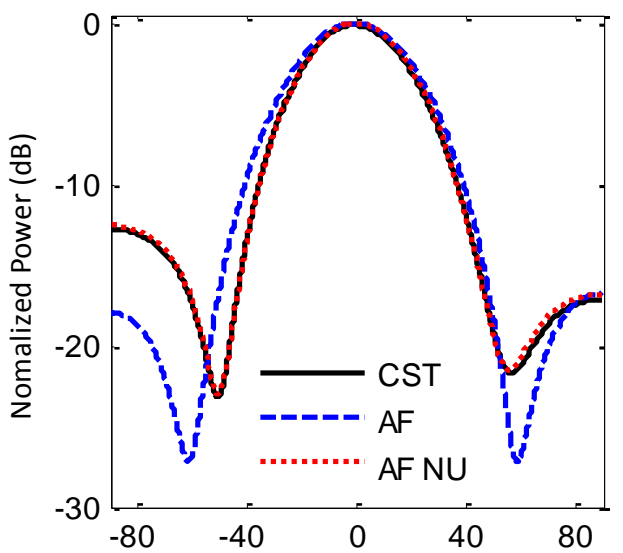

(a)
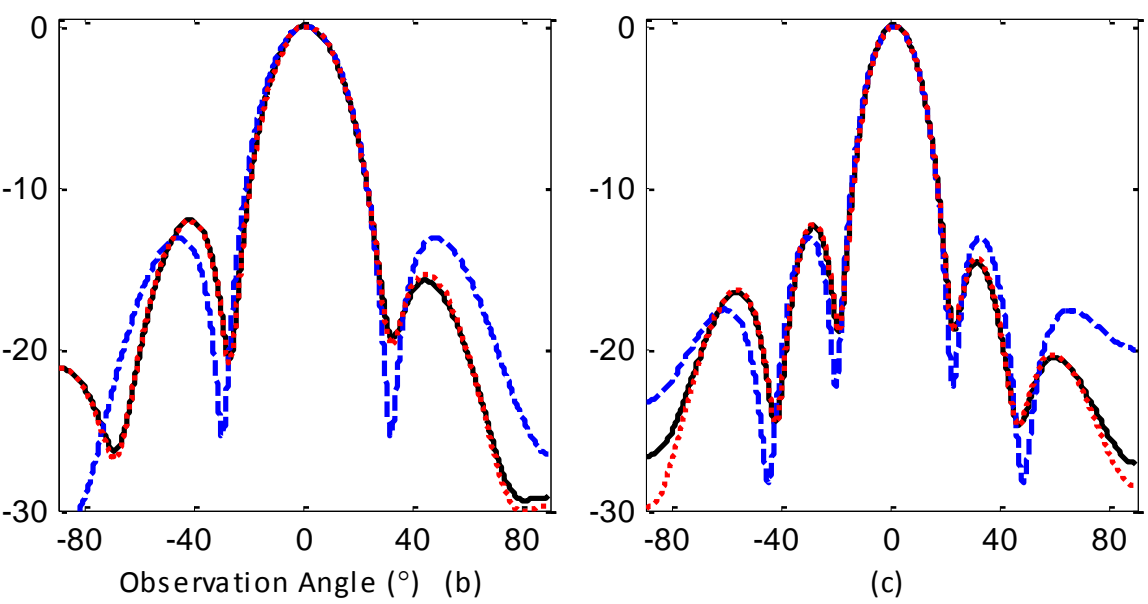

Fig.5 Comparison between non uniform AF, AF and full wave simulation. a) 13 unit cells. b) 22 unit cells. c) 31 unit cells.

multiple solutions with no physical meaning. In order to limit numerical artefact, a weighting function of the form $\cos (q \theta)$ was used. By trying different values of $q$, we obtained a set of solution and we could eliminate the ones too far from the mean tendency.

Examples of distributions obtained are represented on Fig.4 with their corresponding radiation pattern Fig.5. The radiation patterns are matching perfectly with the full-wave simulations. This is natural because of the technique used. Results obtained here are probably not physical but they confirm that a tapering function must be used to model short CRLH LWA. Simulations results also showed that the radiation pattern are robust to some changes in the $\alpha$ and $\beta$ distributions used. Good agreement can still be obtained with distributions which are closer to more plausible physical distributions. The variation of $\beta$ obtained represents a maximum detuning of $\pm 10^{\circ}$ with respect to broadside direction. The extreme values of the distribution observed are singular ones. This is not surprising because they stand for the adaptation cells. The distributions obtained are not regular which is directly related to the technique but they still give a good insight of the numerical values induced by mutual coupling.

Finally, Fig. 6 shows the average values of the non-uniform distributions with respect of the line cell number. The values are not perfectly matching the ones extracted from full- wave simulations but they are following the same tendency.

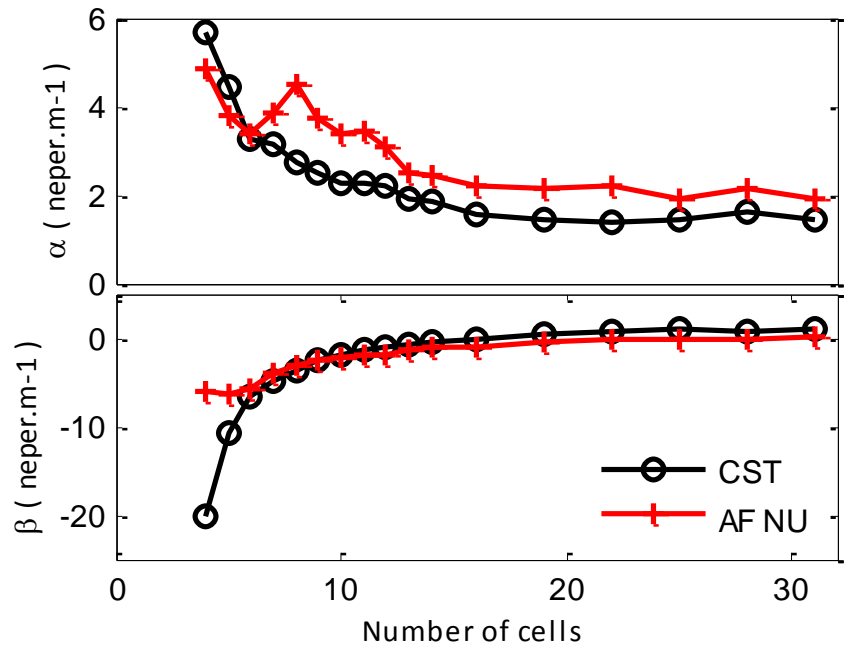

Fig. 6. Comparison between average values of $\alpha \beta$ obtained with non-uniform AF and full wave simulation.

\section{CONCLUSION}

Short uniform CRLH LWA of different lengths have been computed. Differences between full-wave simulations computation with AF approach have been ascribed to the mutual coupling. Better results are achieved when consider non uniform distribution of $\alpha$ and $\beta$. This is a first step towards a more precise and rapid modelling of CRLH radiation patterns.

\section{REFERENCES}

[1] Jackson, D.R.; Caloz, C.; Itoh, T., "Leaky-Wave Antennas," Proceedings of the IEEE, vol.100, no.7, pp.2194,2206, July 2012.

[2] Liu, Lei; Caloz, C.; Itoh, T., "Dominant mode leaky-wave antenna with backfire-to-endfire scanning capability," Electronics Letters , vol.38, no.23, pp.1414,1416, 7 Nov 2002.

[3] Jackson, D.R., "Recent advances in leaky-wave antennas," Electromagnetic Theory (EMTS), Proceedings of 2013 URSI International Symposium on, vol., no., pp.9,12, 20-24 May 2013

[4] Caloz, C.; Itoh, T., "Array factor approach of leaky-wave antennas and application to 1-D/2-D composite right/left-handed (CRLH) structures," Microwave and Wireless Components Letters, IEEE, vol.14, no.6, pp.274,276, June 2004.

[5] Zheng Li; Junhong Wang; Fan Li, "Prediction of radiation patterns of the CRLH leaky-wave antennas by different approaches," Microwave Technology \& Computational Electromagnetics (ICMTCE), 2011 IEEE International Conference on , vol., no., pp.62,65, 22-25 May 2011.

[6] Rance, O.; Lemaître-Auger, P.; Siragusa, R. ; Perret, E. ; "Generalized Array Factor Approach and Design of Tapered Quasi-Uniform Leaky Wave Antenna" under review.

[7] Siragusa, R.; Perret, E.; Lemaitre-Auger, P.; Van Nguyen, H.; Tedjini, S.; Caloz, C., "A Tapered CRLH Interdigital/Stub Leaky-Wave Antenna With Minimized Sidelobe Levels," Antennas and Wireless Propagation Letters, IEEE, vol.11, no., pp.1214,1217, 2012.

[8] A. A. Oliner and D. R. Jackson, "Leaky-Wave Antennas," in Antenna engineering handbook, J. L. Volakis, Ed., 4th ed: McGraw-Hill, 2007.

[9] Steyskal, H.; Herd, J.S., "Mutual coupling compensation in small array antennas," Antennas and Propagation, IEEE Transactions on , vol.38, no.12, pp.1971,1975, Dec 1990.

[10] Yoon-Won Kang; Pozar, David M., "Correction of error in reduced sidelobe synthesis due to mutual coupling," Antennas and Propagation, IEEE Transactions on, vol.33, no.9, pp.1025,1028, Sep 1985. 